\title{
A therapeutic-only versus prophylactic platelet transfusion strategy for preventing bleeding in patients with haematological disorders after chemotherapy or stem cell transplantation
}

\author{
Lise J Estcourt ${ }^{1}$, Gemma L Crighton ${ }^{2}$, Erica M Wood ${ }^{3}$, Simon Stanworth ${ }^{1}$, Marialena \\ Trivella $^{4}$, Carolyn Doree ${ }^{5}$, Alan Tinmouth ${ }^{6}$, and Michael F Murphy ${ }^{7}$ \\ ${ }^{1}$ Haematology/Transfusion Medicine, NHS Blood and Transplant, Oxford, UK \\ ${ }^{2}$ Clinical Haematology, Royal Children's Hospital Melbourne, Melbourne, Australia \\ ${ }^{3}$ Department of Clinical Haematology, Monash University, Melbourne, Australia \\ ${ }^{4}$ Centre for Statistics in Medicine, University of Oxford, Oxford, UK \\ ${ }^{5}$ Systematic Review Initiative, NHS Blood and Transplant, Oxford, UK \\ ${ }^{6}$ Medicine (hematology) and Pathology Centre for Transfusion Research, The Ottawa Health \\ Research Institute and the University of Ottawa, Ottawa, Canada \\ ${ }^{7} \mathrm{NHS}$ Blood and Transplant, John Radcliffe Hospital, Oxford, UK
}

\begin{abstract}
This is the protocol for a review and there is no abstract. The objectives are as follows:

To determine whether a therapeutic-only platelet transfusion policy (platelet transfusions given when patient bleeds) is as effective and safe as a prophylactic platelet transfusion policy (platelet transfusions given to prevent bleeding usually when the platelet count falls below a given trigger level) in patients with haematological disorders undergoing myelosuppressive chemotherapy or stem cell transplantation.
\end{abstract}

Copyright () 2014 The Cochrane Collaboration. Published by John Wiley \& Sons, Ltd.

Contact address: Lise J Estcourt, Haematology/Transfusion Medicine, NHS Blood and Transplant, Level 2, John Radcliffe Hospital, Headington, Oxford, OX3 9BQ, UK. lise.estcourt@nhsbt.nhs.uk. lestcourt@ doctors.org.uk.

Editorial group: Cochrane Haematological Malignancies Group.

Publication status and date: New, published in Issue 3, 2014.

CONTRIBUTIONS OF AUTHORS

Lise Estcourt: protocol development, searching, selection of studies, eligibility and quality assessment, data extraction and analysis and content expert.

Gemma Crighton: protocol development, searching, selection of studies, eligibility and quality assessment, data extraction and analysis and content expert.

Simon Stanworth: protocol development and content expert.

Erica Wood: protocol development and content expert.

Carolyn Doree: protocol development, searching and selection of studies.

Marialena Trivella: protocol development and statistical expert.

Mike Murphy: protocol development and content expert.

Alan Tinmouth: protocol development and content expert.

The previous review Estcourt 2012a has now been split into four separate reviews. 


\section{BACKGROUND}

\section{Description of the condition}

Haematological malignancies account for between $8 \%$ and $9 \%$ of all new cancers reported in the UK and US (CDC 2012; ONS 2012), and their incidence is increasing (11\% to $14 \%$ increase in new cases of lymphoma and myeloma between 1991 to 2001 and 2008 to 2010) (Cancer Research UK 2013). The prevalence of these disorders is also increasing due to increased survival rates (Coleman 2004; Rachet 2009). These improved survival rates are due to the introduction of intensive chemotherapy treatments and use of stem cell transplantation (Burnett 2011; Fielding 2007; Patel 2009). Over 50,000 haematopoietic stem cell transplants (HSCTs) are carried out annually worldwide (Gratwohl 2010), and are used to treat both malignant and non-malignant haematological disorders. Autologous HSCT is the commonest type of HSCT (57\% to 59\%) (Gratwohl 2010; Passweg 2012). However, chemotherapy and stem cell transplantation can lead to prolonged periods of severe thrombocytopenia (De la Serna 2008; Heddle 2009a; Rysler 2010; Stanworth 2013; Wandt 2012).

Platelet transfusions are used in modern clinical practice to prevent and treat bleeding in thrombocytopenic patients with bone marrow failure secondary to chemotherapy or stem cell transplantation. The ready availability of platelet concentrates has undoubtedly made a major contribution in allowing the development of intensive treatment regimens for haematological disorders (malignant and non-malignant) and other malignancies. The first demonstration of the effectiveness of platelet transfusions was performed in 1910 (Duke 1910). However, it was not until the 1970s and 1980s that the use of platelet transfusions became standard treatment for thrombocytopenic patients with bone marrow failure (Blajchman 2008). Alongside changes in supportive care, the routine use of platelet transfusions in patients with haematological disorders since that time has led to a marked decrease in the number of haemorrhagic deaths associated with thrombocytopenia (Slichter 1980). This has resulted in a considerable increase in the demand for platelet concentrates. Currently, platelet concentrates are the second most frequently used blood component. Administration of platelet transfusions to patients with haematological disorders now constitute a significant proportion (up to 67\%) of all platelets issued (Cameron 2007; Greeno 2007; Pendry 2011), and the majority of these (69\%) are given to prevent bleeding (Estcourt 2012b).

Patients can become refractory to platelet transfusions. In an analysis of the TRAP 1997 study data, there was a progressive decrease in the post-transfusion platelet count increments and time interval between transfusions as the number of preceding transfusions increased (Slichter 2005). This effect was seen irrespective of whether or not patients had developed detectable human leukocyte antigen (HLA) antibodies (Slichter 2005).

Platelet transfusions are also associated with adverse events. Mild to moderate reactions to platelet transfusions include rigors, fever, and urticaria (Heddle 2009b). These reactions are not life-threatening but can be extremely distressing for the patient. Rarer, but more serious sequelae include: anaphylaxis; transfusion-transmitted infections; transfusion-related acute lung injury; and immunomodulatory effects (Benson 2009; Blumberg 2009; Bolton-Maggs 
2012; Heddle 2009b; Knowles 2011; Pearce 2011; Popovsky 1985; Silliman 2003; Knowles 2010).

Any strategy that can safely decrease the need for prophylactic platelet transfusions in haematology patients will have significant logistical and financial implications as well as decreasing patients' exposure to the risks of transfusion.

\section{Description of the intervention}

Platelet transfusions have an obvious beneficial effect in the management of active bleeding in patients with haematological malignancy and severe thrombocytopenia. However, questions still remain on how this limited resource should be used to prevent severe and lifethreatening bleeding (Estcourt 2011). Prophylactic platelet transfusions for patients with chemotherapy-induced thrombocytopenia became standard practice following the publication of several, small, randomised controlled trials (RCTs) in the late 1970s and early 1980s (Higby 1974; Murphy 1982; Solomon 1978).

This review does not focus on the absolute need for platelet transfusions in this patient population but will instead focus on whether a prophylactic platelet transfusion policy is required. The standard practice in most haematology units across the developed world is to use prophylactic transfusions, in line with guidelines (Board 2009; BCSH 2003; BCSH 2004; NBA 2012; Schiffer 2001; Slichter 2007; Tinmouth 2007). The experimental intervention will be to give platelet transfusions only when bleeding occurs (therapeuticonly strategy).

\section{How the intervention might work}

\section{Prophylactic platelets versus therapeutic-only platelet transfusions-A}

retrospective review of almost 3000 thrombocytopenic adult patients over a 10-year period showed no relationship between the first morning platelet count, or the lowest platelet count of the day, and the risk of severe or life-threatening bleeding (World Health Organization (WHO) grade 3 to 4 bleeding) (Friedmann 2002). This raised the question as to whether a threshold-defined prophylactic platelet transfusion approach is appropriate. Further large studies have confirmed this finding and also shown no relationship between the morning platelet count and the risk of clinicallysignificant bleeding (WHO grade 2 bleeding) the following day except at very low platelet counts ( $5 \times 10^{9} / \mathrm{L}$ ) (Slichter 2010; Wandt 2012). Further support for the absence of a relationship between the severity of thrombocytopenia and bleeding came from a review of case reports of severe intracranial haemorrhage. No clear evidence was found for an association between the occurrence of major intracranial bleeding and absolute platelet count just prior to the onset of severe bleeding (Stanworth 2005). Thus, the overall benefit of a prophylactic platelet transfusion policy over a policy to use platelets only therapeutically, using a platelet count threshold, has not been established. A recent trial suggested a therapeuticonly platelet transfusion policy might become the new standard of care in selected patients, however the primary endpoint for this study was a reduction in the number of platelet transfusions, rather than a clinical outcome such as bleeding (Wandt 2012). Another large RCT (TOPPS trial) has just been completed and may answer this question (Stanworth 2010; Stanworth 2012). 
Assessment of bleeding-A bleeding assessment is a more clinically-relevant measure of the effect of platelet transfusions than surrogate markers such as platelet count increment.

Any review that uses bleeding as a primary outcome measure needs to assess the way that the trials have recorded bleeding. Unfortunately, the way bleeding has been recorded and assessed has varied markedly between trials (Cook 2004; Estcourt 2013a; Heddle 2003).

Retrospective analysis of bleeding leads to a risk of bias because bleeding events may be missed, and only more severe bleeding is likely to have been documented. Prospective bleeding assessment forms provide more information and are less likely to miss bleeding events. However, different assessors may grade the same bleed differently and it is very difficult to blind the assessor to the intervention.

The majority of trials have used the WHO system, or a modification of it, for grading bleeding (Estcourt 2013a; Koreth 2004; WHO 1979). One limitation of all the scoring systems that are based on the WHO system is that the categories are relatively broad and subjective. This means that a small change in a patient' $\mathrm{s}$ bleeding risk may not be detected. Another limitation is that the modified WHO categories are partially defined by whether a bleeding patient requires a blood transfusion. The threshold for intervention may vary between clinicians and institutions and so the same level of bleeding could be graded differently in different institutions.

The definition of what constitutes clinically-significant bleeding has varied between studies. Although the majority of more recent platelet transfusion studies (Heddle 2009a; Slichter 2010; Stanworth 2012; Wandt 2012) now classify it as WHO grade 2 or above, there has been greater heterogeneity in the past (Cook 2004; Estcourt 2013a; Koreth 2004). The difficulties with assessing and grading bleeding may limit the ability to compare results between studies and this needs to be kept in mind when reviewing the evidence for the effectiveness of prophylactic platelet transfusions.

\section{Why it is important to do this review}

Considerable advances have been made in platelet transfusion therapy in the last 40 years, however three major areas continue to provoke debate:

- Firstly, what is the optimal prophylactic platelet dose to prevent thrombocytopenic bleeding?

- Secondly, which threshold should be used to trigger the transfusion of prophylactic platelets?

- Thirdly, are prophylactic platelet transfusions superior to therapeutic-only platelet transfusions for the prevention and/or control of life-threatening thrombocytopenic bleeding?

The initial formulation of this Cochrane review attempted to answer these questions, but there was insufficient evidence available at the time for any definitive conclusions to be drawn (Stanworth 2004). Although the original review was recently updated (Estcourt 2012a), it is now out-dated because two new large studies have recently been completed 
(Stanworth 2012; Wandt 2012). There is now sufficient additional information regarding these different questions. For clarity and simplicity the review has now been split to answer each question separately.

This review will focus solely on the third question: are prophylactic platelet transfusions superior to therapeutic-only platelet transfusions for the prevention and/or control of lifethreatening thrombocytopenic bleeding?

The other two questions will be assessed by two separate reviews, with an additional third review assessing the use of alternative agents instead of prophylactic platelet transfusions.

Avoiding the need for unnecessary prophylactic platelet transfusions in haematology patients will have significant logistical and financial implications for national health services as well as decreasing patients' exposure to the risks of transfusion. This knowledge is perhaps even more important in the development of platelet transfusion strategies in the developing world, where access to blood components is much more limited (Verma 2009).

This review will not assess whether there are any differences in the efficacy of apheresis versus whole-blood derived platelet products, the efficacy of pathogen-reduced platelet components, the efficacy of HLA-matched versus random donor platelets, or differences between $\mathrm{ABO}$ identical and $\mathrm{ABO}$ non-identical platelet transfusions. These topics have been covered by recent systematic reviews (Butler 2013; Heddle 2008; Pavenski 2013; Shehata 2009).

\section{OBJECTIVES}

To determine whether a therapeutic-only platelet transfusion policy (platelet transfusions given when patient bleeds) is as effective and safe as a prophylactic platelet transfusion policy (platelet transfusions given to prevent bleeding usually when the platelet count falls below a given trigger level) in patients with haematological disorders undergoing myelosuppressive chemotherapy or stem cell transplantation.

\section{METHODS}

\section{Criteria for considering studies for this review}

Types of studies-We will include randomised controlled trials (RCTs). There will be no restrictions on language or publication status.

Types of participants-Patients with haematological disorders receiving treatment with myelosuppressive chemotherapy and/or stem cell transplantation. We will include people of all ages and we will include both inpatients and outpatients.

If trials consist of mixed populations of patients, e.g. patients with diagnoses of solid tumours, only data from the haematological subgroups will be used. If subgroup data for haematological patients are not provided (after contacting the authors of the trial), the trial will be excluded if fewer than $80 \%$ of participants have a haematological disorder. Any patients who are not being treated with intensive chemotherapy or a stem cell transplant will 
be excluded. We will include patients with non-malignant haematological disorders (e.g. aplastic anaemia, congenital bone marrow failure syndromes) that are being treated with an allogeneic stem cell transplant.

Types of interventions-Patients in both treatment arms will receive transfusions of platelet concentrates, prepared either from individual units of whole blood or by apheresis to treat bleeding (therapeutic platelet transfusions). Patients in the control arm will also receive prophylactic platelet transfusions. Prophylactic platelet transfusions are typically given when the platelet count falls below a given trigger level. There will be no restriction on the dose, frequency, type of platelet component, or transfusion trigger of the platelet transfusions but we will take this information into account in the analysis, where available. We will include the following comparisons:

- Therapeutic-only platelet transfusions (on-demand triggered by bleeding) versus prophylactic platelet transfusions

- Placebo versus prophylactic platelet transfusions

\section{Types of outcome measures}

Primary outcomes: Number and severity of bleeding episodes within 30 days from the start of the study:

- The number of patients with at least one bleeding episode.

- The total number of days on which bleeding occurred per patient.

- The number of patients with at least one episode of severe or life-threatening bleeding.

- Time to first bleeding episode from the start of the study.

\section{Secondary outcomes:}

- Mortality (all-causes, secondary to bleeding, and secondary to infection) within 30 days and 90 days from the start of the study.

- Number of platelet transfusions per patient and number of platelet components per patient within 30 days from the start of the study.

- Number of red cell transfusions per patient and number of red cell components per patient within 30 days from the start of the study.

- Platelet transfusion interval within 30 days from the start of the study.

- Proportion of patients requiring additional interventions to stop bleeding (surgical, medical e.g. tranexamic acid, other blood products e.g. fresh frozen plasma (FFP), cryoprecipitate) within 30 days from the start of the study.

- Overall survival within 30 days, 90 days, and 180 days from the start of the study.

- Proportion of patients achieving complete remission within 30 days and 90 days from the start of the study. 
- The total time in hospital within 30 days from the start of the study.

- Adverse effects of treatments (transfusion reactions, thromboembolism, transfusion-transmitted infection, development of platelet antibodies, development of platelet refractoriness) within 30 days and 90 days from the start of the study.

- Quality of life, as defined by the individual studies.

We will express all primary and secondary outcomes in the formats defined in the Measures of treatment effect section of this protocol if data are available. Two of our outcomes are of special note as we expect them to be only narrative reports. Firstly, assessment of quality of life will use the study' $s$ own measure as there is no definitive patient reported outcome measure for this patient group (Estcourt 2013b). Secondly, the platelet transfusion interval can be calculated in many different ways and it is unlikely that the exact methodology will be reported sufficiently to allow us to combine the data.

\section{Search methods for identification of studies}

The Systematic Review Initiative (SRI) Information Specialist (CD) formulated new search strategies in collaboration with the Cochrane Haematological Malignancies Review Group based on those used in previous versions of this review (Estcourt 2012a; Stanworth 2004).

\section{Electronic searches}

Bibliographic databases: We will search for randomised controlled trials in the following databases:

- CENTRAL (The Cochrane Library) (Appendix 1)

- MEDLINE (Ovid, 1946 to the present) (Appendix 2)

- PubMed (epublications only) (Appendix 3)

- $\quad$ Embase (Ovid, 1974 to the present) (Appendix 4)

- CINAHL (EBSCOhost, 1982 to the present) (Appendix 5)

- UKBTS/SRI Transfusion Evidence Library (www.transfusionevidencelibrary.com) (1980 to the present) (Appendix 6)

- Web of Science: Conference Proceedings Citation Index-Science (CPCI-S) (Thomson Reuters, 1990 to the present) (Appendix 7)

- Lilacs (BIREME/PAHO/WHO, 1982 to the present) (Appendix 8)

- IndMed (ICMR-NIC, 1985 to the present) (Appendix 9)

- KoreaMed (KAMJE, 1997 to the present) (Appendix 10)

- PakMediNet (2001 to the present) (Appendix 10)

Searches will be updated from the original search in January 2002 (Stanworth 2004) and the updated search on 10th November 2011 (Estcourt 2012a). Searches in MEDLINE, Embase and CINAHL will be combined with adaptations of the Cochrane RCT search filters, as 
detailed in the Cochrane Handbook for Systematic Reviews of Interventions (Lefebvre 2011).

Databases of ongoing trials: We will also search ClinicalTrials.gov (http://

clinicaltrials.gov/ct2/search) (Appendix 11), the WHO International Clinical Trials Registry (ICTRP) (http://apps.who.int/trialsearch/) (Appendix 11), the ISRCTN Register (http:// www.controlled-trials.com/isrctn/) (Appendix 12), the EU Clinical Trials Register (https:// www.clinicaltrialsregister.eu/ctr-search) (Appendix 12) and the Hong Kong Clinical Trials Register (http://www.hkclinicaltrials.com/) (Appendix 13) in order to identify ongoing trials.

All new search strategies are presented as indicated in Appendices 1-13. Search strategies for both the original (2002) and update (2011) searches are presented in Appendix 14.

\section{Searching other resources}

Handsearching of reference lists: We will check references of all included trials, relevant review articles and current treatment guidelines for further literature. These searches will be limited to the 'first generation' reference lists.

Personal contacts: We will contact authors of relevant studies, study groups and experts worldwide known to be active in the field for unpublished material or further information on ongoing studies.

\section{Data collection and analysis}

Selection of studies-The selection of studies will be updated from the selection of studies performed for the previous version of this review (Estcourt 2012a).

Two independent review authors (GC, LE) will initially screen all electronically-derived citations and abstracts of papers identified by the review search strategy for relevance. Studies clearly irrelevant will be excluded at this stage.

The full texts of all potentially-relevant trials will then be formally assessed for eligibility by two independent review authors (GC, LE) against the criteria outlined above. All disagreements will be resolved by discussion with a third review author (SS). Further information will be sought from study authors if the article contains insufficient data to make a decision about eligibility. A study eligibility form will be designed for trials of platelet transfusion to help in the assessment of relevance, which will include ascertaining whether the participants had haematological disorders, and whether the two groups could be defined in the trial on the basis of a therapeutic-only versus prophylactic platelet transfusion strategy. The reasons why potentially-relevant studies failed to meet the eligibility criteria will be recorded.

Data extraction and management-The data extraction will be updated from the data extraction performed for the previous version of this review (Estcourt 2012a). This will include data extraction for all new studies that have been included since the previous review and also for all review outcomes that were not part of the previous review (e.g. platelet transfusion interval, quality of life). 
Two review authors (GC, LE) will conduct data extraction according to the guidelines proposed by the Cochrane Collaboration (Higgins 2011a). Potential disagreements between the review authors will be resolved by consensus. The review authors will not be blinded to names of authors, institutions, journals, or the outcomes of the trials. The data extraction forms have been piloted in the previous version of this review (Estcourt 2012a). Due to minor changes in the format the forms will piloted on a further study, thereafter the two authors (GC, LE) will extract data independently for all the studies. The following data will be extracted:

General information: Review author's name, date of data extraction, study ID, reference manager number, first author of study, author's contact address (if available), citation of paper, objectives of the trial.

Trial details: Trial design, location, setting, sample size, power calculation, treatment allocation, randomisation, blinding, inclusion and exclusion criteria, reasons for exclusion, comparability of groups, length of follow up, stratification, stopping rules described, statistical analysis, results, conclusion, and funding.

Characteristics of participants: Age, gender, ethnicity, total number recruited, total number randomised, total number analysed, types of haematological disease, lost to followup numbers, drop outs (percentage in each arm) with reasons, protocol violations, previous treatments, current treatment, prognostic factors.

Interventions: Experimental and control interventions, type of platelet given, timing of intervention, dosage of platelet given, compliance to interventions, additional interventions given especially in relation to red cell transfusions, any differences between interventions.

Assessment of bias: Sequence generation, allocation concealment, blinding (participants, personnel, and outcome assessors), incomplete outcome data, selective outcome reporting, other sources of bias.

Outcomes measured: Number and severity of bleeding episodes. Mortality (all causes), and mortality due to bleeding. Disease-free survival. Proportion of patients achieving complete remission. Time in hospital. Number of platelet transfusions and platelet components. Number of red cell transfusions and red cell components. Platelet transfusion interval. Proportion of patients requiring additional interventions to stop bleeding (surgical, medical e.g. tranexamic acid, other blood products e.g. fresh frozen plasma (FFP), cryoprecipitate). Quality of life. Adverse effects of treatments (e.g. transfusion reactions, thromboembolism, transfusion-transmitted infection, development of platelet antibodies or platelet refractoriness). Both full-text versions and abstracts will be used to retrieve the data. Publications reporting on more than one trial will be extracted using one data extraction form for each trial. Trials reported in more than one publication will be extracted on one form only. Where these sources do not provide sufficient information, we will contact authors, study groups or companies for additional details. 
Data entry into software will be done by one review author and will be checked for accuracy by a second review author.

\section{Assessment of risk of bias in included studies}

The ' Risk of bias' assessment will be updated from the 'Risk of bias' assessment performed for the previous version of this review (Estcourt 2012a).

Two review authors (GC, LE) will assess all newly-included studies for possible risk of bias (as described in the Cochrane Handbook (Higgins 2011c)). The assessment will include information about the design, conduct and analysis of the trial. Each criterion will be evaluated on a three-point scale: low risk of bias, high risk of bias, or unclear. To assess risk of bias, the following questions will be included in the 'Risk of bias' table for each included study:

- Was the allocation sequence adequately generated?

- Was allocation adequately concealed?

- Was knowledge of the allocated intervention adequately prevented during the study (including an assessment of blinding of participants, personnel, and outcome assessors)?

- Were incomplete outcome data adequately addressed (for every outcome separately)?

- Are reports of the study free of selective outcome reporting?

- Was the study apparently free of other problems that could put it at risk of bias?

\section{Measures of treatment effect}

For dichotomous outcomes the number of outcomes in treatment and control groups will be recorded and the treatment effect measures across individual studies will be estimated as the relative effect measures (relative risk (RR) with 95\% confidence intervals (CIs)).

For continuous outcomes, the mean and standard deviations will be recorded. For continuous outcomes measured using the same scale the effect measure will be the mean difference (MD) with 95\% CIs, or the standardised mean difference (SMD) for outcomes measured using different scales.

For time-to-event outcomes we will extract the hazard ratio (HR) from published data according to Parmar 1998 and Tierney 2007. If appropriate, the number needed to treat to benefit (NNTB) with CIs and the number needed to treat to harm (NNTH) with CIs will be reported.

If the data available cannot be reported in any of the formats described above a narrative report will be performed. 


\section{Dealing with missing data}

Missing data will be dealt with according to the recommendations in the Cochrane Handbook (Higgins 2011b). We will contact authors in order to obtain information that is missing or unclear in the published report.

In trials that include patients with haematological disorders as well as patients with solid tumours or non-malignant haematological disorders, data will be extracted for the haematology subgroup that is receiving chemotherapy or a stem cell transplantation from the general trial data. If this cannot be done the author will be contacted.

Within an outcome, when there are missing data, the preferred analysis will be an intentionto-treat analysis (ITT). The number of patients lost to follow-up will be recorded for each trial.

\section{Assessment of heterogeneity}

If studies are considered sufficiently homogenous in their study design, we will conduct a meta-analysis and assess the statistical heterogeneity (Deeks 2011). Statistical heterogeneity of treatment effects between trials will be assessed using a $\mathrm{Chi}^{2}$ test with a significance level at $\mathrm{P}<0.1$. The $\mathrm{I}^{2}$ statistic will be used to quantify possible heterogeneity $\left(\mathrm{I}^{2}>50 \%\right.$ moderate heterogeneity, $\mathrm{I}^{2}>80 \%$ considerable heterogeneity). Potential causes of heterogeneity will be explored by sensitivity and subgroup analyses if possible.

\section{Assessment of reporting biases}

We will explore meta-analyses with at least 10 trials for potential publication bias (small trial bias) by generating a funnel plot and statistically test using a linear regression test. We will consider a $\mathrm{P}$ value of less than 0.1 significant for this test (Sterne 2011).

\section{Data synthesis}

Analyses will be performed according to the recommendations of the Cochrane Collaboration (Deeks 2011). Aggregated data will be used for analysis. For statistical analysis, data will be entered into Review Manager 2012.

Where meta-analysis is feasible, the fixed-effect model will be used for pooling the data. The Mantel-Haenszel method will be used for dichotomous outcomes, and the inverse variance method for continuous outcomes. The generic inverse variance method will be employed for time-to-event outcomes.

We will use the random-effects model for sensitivity analyses as part of the exploration of heterogeneity. If heterogeneity, as expressed by the $\mathrm{I}^{2}$, is found to be above $50 \%$, both the fixed-effect and random-effects models will be reported. If heterogeneity is found to be above $80 \%$, we will not perform a meta-analysis and results will be commented on as a narrative.

GRADEprofiler will be used to create 'Summary of findings' tables as suggested in the Cochrane Handbook (Schünemann 2011). This will include the number and severity of bleeding episodes within 30 days from the start of the study (number of patients with at least 
one bleeding episode; number of days on which bleeding occurred; number of patients with severe or life-threatening bleeding; time to first bleeding episode), number of platelet transfusions within 30 days from the start of the study, overall mortality at 30 days, and quality of life.

\section{Subgroup analysis and investigation of heterogeneity}

Two subgroup analyses have been pre-specified prior to the previous version of this review; these are fever and patients' diagnostic and treatment subgroups. We will consider performing subgroup analysis on the following characteristics, if appropriate:

- Presence of fever $\left(>38^{\circ} \mathrm{C}\right)$

- Underlying disease

- Type of treatment (autologous HSCT, allogeneic HSCT, or chemotherapy alone)

- Age of the patient (paediatric, adults, older adults (> 60 years))

Meta-regression will be performed if subgroups contain more than 10 studies (Deeks 2011). Differences between subgroups will be compared using a random-effects model when the two subgroups are independent following the guidance in Chapter 9 of the Cochrane Handbook (Deeks 2011). If this is not possible then differences will be commented on as a narrative.

Investigation of heterogeneity between studies will also include, if appropriate:

- Age of the study (as the type of platelet component has changed over the last 40 years)

- Different platelet component doses

- Different prophylactic platelet transfusion thresholds

\section{Sensitivity analysis}

Robustness of the overall results will be assessed by sensitivity analysis with respect to those trials deemed at high risk of bias. For dichotomous data, the influence of participant drop-out will be assessed by analysing separately RCTs with less than $20 \%$ dropout, RCTs with $20 \%$ to $50 \%$ drop-out and RCTs with greater than 50\% drop-out.

We will use the random-effects model for sensitivity analyses as part of the exploration of heterogeneity.

\section{ACKNOWLEDGEMENTS}

We thank the editorial base of the Cochrane Haematological Malignancies Review Group.

We thank the authors on the previous reviews: S Brunskill; S Hopewell; N Heddle; C Hyde; P Rebulla.

\section{SOURCES OF SUPPORT}

Internal sources

- $\quad$ NHS Blood and Transplant, Research and Development, UK. 
To fund the work of the Systematic Review Initiative (SRI)

External sources

- German Ministry of Education and Research (BMBF) towards support of the Cochrane Haematological Malignancies Group, Germany.

For Editorial Support

\section{Appendix 1. CENTRAL (The Cochrane Library) 2013 search strategy}

\#1 MeSH descriptor: [Blood Platelets] explode all trees

\#2 (platelet* or thrombocyte*):ti

\#3 \#1 or \#2

\#4 MeSH descriptor: [Blood Transfusion] explode all trees

\#5 transfus*:ti

\#6 \#4 or \#5

\#7 \#3 and \#6

\#8 MeSH descriptor: [Platelet Transfusion] explode all trees

\#9 MeSH descriptor: [Plateletpheresis] explode all trees

\#10 ((platelet* or thrombocyte*) near/5 (prophyla* or transfus* or infus* or administ* or requir* or need* or product or products or component* or concentrate* or apheres* or pooled or single donor or random donor))

\#11 thrombocytopheres* or plateletpheres*

\#12 ((platelet* or thrombocyte*) near/5 (protocol* or trigger* or threshold* or schedul* or dose* or dosing or usage or utilisation or utilization))

$\# 13 \# 7$ or \#8 or \#9 or \#10 or \#11 or \#12

\#14 MeSH descriptor: [Hematologic Neoplasms] explode all trees

\#15 MeSH descriptor: [Leukemia] explode all trees

\#16 MeSH descriptor: [Lymphoma] explode all trees

\#17 MeSH descriptor: [Multiple Myeloma] explode all trees

\#18 MeSH descriptor: [Anemia, Aplastic] explode all trees

\#19 MeSH descriptor: [Bone Marrow Diseases] explode all trees

\#20 MeSH descriptor: [Thrombocytopenia] explode all trees 
\#21 (thrombocytope* or leukemi* or leukaemi* or lymphoma* or aplastic anemia or aplastic anaemia or myelodysplas* or myeloproliferat* or multiple myeloma or plasma cell myeloma or thrombocythemi* or thrombocythaemi* or polycythemi* or polycythaemi* or myelofibros* or AML or CLL or CML or Hodgkin*)

\#22 ((haematolog* or hematolog* or blood or red cell* or white cell* or lymph* or marrow or platelet*) near/3 (malignan* or oncolog* or cancer* or neoplasm*))

\#23 MeSH descriptor: [Antineoplastic Agents] explode all trees

\#24 MeSH descriptor: [Stem Cell Transplantation] explode all trees

\#25 MeSH descriptor: [Bone Marrow Transplantation] this term only

\#26 MeSH descriptor: [Radiotherapy] explode all trees

\#27 (chemotherap* or radiotherap* or chemoradiotherap* or chemo-radiotherap* or stem cell* or bone marrow transplant*)

\#28 ((haematolog* or hematolog* or hemato-oncolog* or haemato-oncolog*) near/2 patients)

\#29 (malignan* or oncolog* or cancer*):ti

$\# 30 \# 14$ or \#15 or \#16 or \#17 or \#18 or \#19 or \#20 or \#21 or \#22 or \#23 or \#24 or \#25 or \#26 or \#27 or \#28 or \#29

$\# 31 \# 13$ and \#30

\section{Appendix 2. MEDLINE (Ovid) search strategy (Nov 2011-2013)}

\section{BLOOD PLATELETS/}

2. (platelet* or thrombocyte*).ti.

3. 1 or 2

4. $\operatorname{exp~BLOOD~TRANSFUSION/~}$

5. transfus*.ti.

6. 4 or 5

7. 3 and 6

8. PLATELET TRANSFUSION/

9. PLATELETPHERESIS/

10. ((platelet* or thrombocyte*) adj5 (prophyla* or transfus* or infus* or administ* or requir* or need* or product* or component* or concentrate* or apheres* or pooled or single donor or random donor)).tw.

11. (thrombocytopheres* or plateletpheres*).tw. 
12. ((platelet* or thrombocyte*) adj5 (protocol* or trigger* or threshold* or schedul* or dose* or dosing or usage or utili?ation)).tw.

13. or $/ 7-12$

14. exp Hematologic Neoplasms/

15. exp Leukemia/or exp Lymphoma/

16. exp Multiple Myeloma/

17. exp Anemia, Aplastic/

18. exp Bone Marrow Diseases/

19. $\exp$ Thrombocytopenia/

20. (thrombocytopeni* or thrombocytopaeni* or leukemia or leukaemia or lymphoma* or aplastic anemia or aplastic anaemia or myelodysplas* or myeloproliferat* or multiple myeloma or plasma cell myeloma or thrombocythemi* or thrombocythaemi* or polycythemi* or polycythaemi* or myelofibros* or AML or CLL or CML or Hodgkin*).tw.

21. ((haematolog* or hematolog* or blood or red cell* or white cell* or lymph* or marrow or platelet*) adj3 (malignan* or oncolog* or cancer* or neoplasm*)).tw.

22. exp Antineoplastic Agents/

23. exp Stem Cell Transplantation/or Bone Marrow Transplantation/or exp Radiotherapy/

24. (chemotherap* or radiotherap* or chemoradiotherap* or chemo-radiotherap* or stem cell* or bone marrow transplant*).tw.

25. ((haematolog* or hematolog* or haemato-oncolog* or hemato-oncolog*) adj2 patients).tw.

26. (malignan* or oncolog* or cancer*).ti.

27. or/ $14-26$

28. 13 and 27

\section{Appendix 3. PubMed search strategy (epublications only)}

\#1 ((platelet* OR thrombocyte*) AND (prophyla* OR transfus* OR infus* OR administ* OR requir* OR need* OR product OR products OR component* OR concentrate* OR apheres* OR pooled OR single donor OR random donor OR protocol* OR trigger* OR threshold* OR schedul* OR dose OR doses OR dosing OR usage OR utilisation OR utilization))

\#2 thrombocytopheres* OR plateletpheres*

\#3 \#1 OR \#2 
\#4 (thrombocytop* OR leukemi* OR leukaemi* OR lymphoma* OR aplastic anemia OR aplastic anaemia OR myelodysplas* OR myeloproliferat* OR multiple myeloma OR plasma cell myeloma OR thrombocythemi* OR thrombocythaemi* OR polycythemi* OR polycythaemi* OR myelofibros* OR Hodgkin*)

\#5 ((haematolog* OR hematolog* OR blood OR red cell* OR white cell* OR lymphom* OR marrow OR platelet*) AND (malignan* OR oncolog* OR cancer OR cancers OR neoplasm*))

\#6 \#4 OR \#5

\#7 \#3 AND \#6

\#8 (random* OR blind* OR control group* OR placebo OR controlled trial OR controlled study OR trials OR systematic review OR meta-analysis OR metaanalysis OR literature OR medline OR cochrane OR embase) AND (publisher[sb] NOT pubstatusnihms)

\#9 \#7 AND \#8

\section{Appendix 4. EMBASE (Ovid) search strategy (Nov 2011-2013)}

1. Thrombocyte/

2. (platelet* or thrombocyte*).ti.

3. 1 or 2

4. Blood Transfusion/

5. transfus*.ti.

6. 4 or 5

7. 3 and 6

8. Thrombocyte Transfusion/

9. Thrombocytopheresis/

10. ((platelet* or thrombocyte*) adj5 (prophyla* or transfus* or infus* or administ* or requir* or need* or product* or component* or concentrate* or apheres* or pooled or single donor or random donor)).tw.

11. (thrombocytopheres* or plateletpheres*).tw.

12. ((platelet* or thrombocyte*) adj5 (protocol* or trigger* or threshold* or schedul* or dose* or dosing or usage or utili?ation)).tw.

13. or $/ 7-12$

14. Hematologic Malignancy/

15. Lymphoma/

16. NonHodgkin Lymphoma/ 


\section{Hodgkin Disease/}

18. exp Myeloproliferative Disorder/

19. exp Aplastic Anemia/

20. exp Thrombocytopenia/

21. (thrombocytopeni* or thrombocytopaeni* or leukemia or leukaemia or lymphoma* or aplastic anemia or aplastic anaemia or myelodysplas* or myeloproliferat* or multiple myeloma or plasma cell myeloma or thrombocythemi* or thrombocythaemi* or polycythemi* or polycythaemi* or myelofibros* or AML or CLL or CML or Hodgkin*).tw.

22. ((haematolog* or hematolog* or blood or red cell* or white cell* or lymph* or marrow or platelet*) adj3 (malignan* or oncolog* or cancer* or neoplasm*)).tw.

23. exp Chemotherapy/

24. exp Stem Cell Transplantation/

25. exp Bone Marrow Transplantation/

26. exp Radiotherapy/

27. (chemotherap* or radiotherap* or chemoradiotherap* or chemo-radiotherap* or stem cell* or bone marrow transplant* or rituximab).tw.

28. ((haematolog* or hematolog*) adj2 patients).tw.

29. (malignan* or oncolog* or cancer*).ti.

30. or $/ 14-29$

31. 13 and 30

\section{Appendix 5. CINAHL (EBSCOhost) search strategy (Nov 2011-2013)}

S1 (MH "Blood Platelets")

S2 TI (platelet* or thrombocyte*)

S3 S1 OR S2

S4 (MH "BLOOD TRANSFUSION+")

S5 TI transfus*

S6 S4 or S5

S7 S3 and S6

S8 (MH "PLATELET TRANSFUSION")

S9 (MH PLATELETPHERESIS) 
S10 ((platelet* or thrombocyte*) N5 (prophyla* or transfus* or infus* or administ* or requir* or need* or product* or component* or concentrate* or apheres* or pooled or single donor or random donor))

S11 (thrombocytopheres* or plateletpheres*)

S12 ((platelet* or thrombocyte*) N5 (protocol* or trigger* or threshold* or schedul* or dose* or dosing or usage or utili?ation))

\section{S13 S8 OR S9 OR S10 OR S11 OR S12}

S14 (MH "Hematologic Neoplasms+")

S15 (MH Leukemia+)

S16 (MH Lymphoma+)

S17 (MH "Multiple Myeloma+")

S18 (MH “Anemia, Aplastic+”)

S19 (MH “Bone Marrow Diseasest”)

S20 (MH Thrombocytopenia+)

S21 (thrombocytopeni* or thrombocytopaeni* or leukemia or leukaemia or lymphoma* or aplastic anemia or aplastic anaemia or myelodysplas* or myeloproliferat* or multiple myeloma or plasma cell myeloma or thrombocythemi* or thrombocythaemi* or polycythemi* or polycythaemi* or myelofibros* or AML or CLL or CML or Hodgkin*)

S22 ((haematolog* or hematolog* or blood or red cell* or white cell* or lymph* or marrow or platelet*) N3 (malignan* or oncolog* or cancer* or neoplasm*))

S23 (MH “Antineoplastic Agents+")

S24 (MH "Hematopoietic Stem Cell Transplantation")

S25 (MH "Bone Marrow Transplantation")

S26 (MH Radiotherapy+)

S27 (chemotherap* or radiotherap* or chemoradiotherap* or chemo-radiotherap* or stem cell* or bone marrow transplant*)

S28 ((haematolog* or hematolog* or haemato-oncolog* or hemato-oncolog*) N2 patients)

S29 TI (malignan* or oncolog* or cancer*)

S30 S14 OR S15 OR S16 OR S17 OR S18 OR S19 OR S20 OR S21 OR S22 OR S23 OR S24 OR S25 OR S26 OR S27 OR S28 OR S29 
S31 S13 and S30

\section{Appendix 6. TRANSFUSION EVIDENCE LIBRARY search strategy (2013)}

\#1 ((platelet* OR thrombocyte*) AND (prophyla* OR transfus* OR infus* OR administ* OR requir* OR need* OR product OR products OR component* OR concentrate* OR apheres* OR pooled OR single donor OR random donor OR protocol* OR trigger* OR threshold* OR schedul* OR dose OR doses OR dosing OR usage OR utilisation OR utilization))

\#2 thrombocytopheres* OR plateletpheres*

\#3 \#1 OR \#2

\#4 (thrombocytop* OR leukemi* OR leukaemi* OR lymphoma* OR aplastic anemia OR aplastic anaemia OR myelodysplas* OR myeloproliferat* OR multiple myeloma OR plasma cell myeloma OR thrombocythemi* OR thrombocythaemi* OR polycythemi* OR polycythaemi* OR myelofibros* OR Hodgkin*)

\#5 ((haematolog* OR hematolog* OR blood OR red cell* OR white cell* OR lymphom* OR marrow OR platelet*) AND (malignan* OR oncolog* OR cancer OR cancers OR neoplasm*))

\#6 \#4 OR \#5

\#7 \#3 AND \#6

\section{Appendix 7. Web of Science (CPCI-S) search strategy (2013)}

((platelet* AND (prophyla* OR transfus* OR products OR component* OR concentrate* OR apheres* OR pooled OR single donor OR random donor OR protocol* OR trigger* OR threshold*)) AND (thrombocytop* OR leukemi* OR leukaemi* OR lymphoma* OR aplastic OR myelodysplas* OR myeloproliferat* OR myeloma OR thrombocythemi* OR thrombocythaemi* OR polycythemi* OR polycythaemi* OR myelofibros* OR hodgkin* OR haematological OR hematological)) [in Title]

AND (randomized OR randomised OR randomly) [in Title]

\section{Appendix 8. LILACS search strategy (2013)}

((platelet* AND (prophyla* OR transfus* OR products OR component* OR concentrate* OR apheres* OR pooled OR single donor OR random donor OR protocol* OR trigger* OR threshold*)) AND (thrombocytop* OR leukemi* OR leukaemi* OR lymphoma* OR aplastic OR myelodysplas* OR myeloproliferat* OR myeloma OR thrombocythemi* OR thrombocythaemi* OR polycythemi* OR polycythaemi* OR myelofibros* OR hodgkin* OR haematological OR hematological)) AND db:(“LILACS") AND type_of_study: (“clinical_trials" OR "systematic_reviews") 


\title{
Appendix 9. INDMED search strategy (2013)
}

(platelet OR platelets OR thrombocyte\$ OR thrombocytopheres\$ OR plateletpheres\$) AND (thrombocytop\$ OR leukemi\$ OR leukaemi\$ OR lymphoma \$ OR aplastic OR myelodysplas \$ OR myeloproliferat\$ OR myeloma OR thrombocythemi\$ OR thrombocythaemi\$ OR polycyth\$ OR myelofibros\$ OR Hodgkin\$ OR haematological OR hematological OR hematopoietic OR haematopoietic) AND (random\$ OR blind\$ OR trial\$ OR control\$)

\author{
Appendix 10. KoreaMed \& PakMediNet search strategy (2013) \\ platelet*[ALL] AND “Randomized Controlled Trial” [PT] \\ thrombocyt*[ALL] AND "Randomized Controlled Trial" [PT]
}

\section{Appendix 11. ClinicalTrials.gov \& ICTRP search strategy (2013) \\ Search Terms/Title: randomized OR randomised \\ Conditions: hematological neoplasm OR hematological malignancies OR leukemia OR lymphoma OR thrombocytopenia OR multiple myeloma OR aplastic anemia OR thrombocythemia OR polycythemia OR myelofibrosis OR hodgkins disease Intervention: platelets OR platelet transfusion}

\section{Appendix 12. ISRCTN \& EU Clinical Trials Register search strategy (2013) (hematological OR haematological OR leukemi* OR leukaemi* OR lymphoma OR thrombocytopeni* OR myeloma OR aplastic OR thrombocythemia OR polycythemia OR myelofibrosis OR hodgkin*) AND platelet* transfus* AND random*}

\author{
Appendix 13. Hong Kong Clinical Trials Register search strategy (2013) \\ Disease Group: Blood and blood-forming organs \\ Title: randomized OR randomised
}

\section{Appendix 14. Previous searches: original (Jan 2002) \& update (Nov 2011) search strategies}

\section{CENTRAL search strategy (Issue 4, 2011)}

\#1 MeSH descriptor Blood Platelets explode all trees

\#2 platelet* or thrombocyte*

\#3 (\#1 OR \#2)

\#4 MeSH descriptor Blood Transfusion explode all trees

\#5 transfus* 
\#6 (\#4 OR \#5)

\#7 (\#3 AND \#6)

\#8 MeSH descriptor Platelet Transfusion explode all trees

\#9 (platelet* or thrombocyte*) NEAR/5 (transfus* or infus* or administ* or requir*)

\#10 (\#7 OR \#8 OR \#9)

\#11 prophylactic* or prophylax* or prevent*

\#12 (\#10 AND \#11)

\section{MEDLINE (Ovid) search strategy (Jan 2002 - Nov 2011)}

1. BLOOD PLATELETS/

2. (platelet* or thrombocyte*).tw.

3. 1 or 2

4. $\exp$ BLOOD TRANSFUSION/

5. transfus*.tw.

6. 4 or 5

7. 3 and 6

8. PLATELET TRANSFUSION/

9. ((platelet* or thrombocyte*) adj5 (transfus* or infus* or administ* or requir*)).tw.

10. or/7-9

11. (prophylactic* or prophylax* or prevent*).tw.

12. 10 and 11

\section{Embase (Ovid) search strategy (Jan 2002 - Nov 2011)}

\section{THROMBOCYTE/}

2. (platelet* or thrombocyte*).tw.

3. 1 or 2

4. $\exp$ BLOOD TRANSFUSION/

5. transfus*.tw.

6. 4 or 5

7. 3 and 6

8. THROMBOCYTE TRANSFUSION/ 
9. ((platelet* or thrombocyte*) adj5 (transfus* or infus* or administ* or requir*)).tw.

10. or $/ 7-9$

11. (prophylactic* or prophylax* or prevent*).tw.

12. 10 and 11

\section{CINAHL (NHS Evidence) search strategy (Jan 2002 - Nov 2011)}

1. BLOOD PLATELETS/

2. (platelet* or thrombocyte*).ti,ab

3. 1 or 2

4. $\exp$ BLOOD TRANSFUSION/

5. transfus*.ti,ab

6. 4 or 5

7. 3 and 6

8. PLATELET TRANSFUSION/

9. ((platelet* adj5 transfus*) or (platelet* adj5 infus*) or (platelet* adj5 administ*) or (platelet* adj5 requir*)).ti,ab

10. ((thrombocyte* adj5 transfus*) or (thrombocyte* adj5 infus*) or (thrombocyte* adj5 administ*) or (thrombocyte* adj5 requir*)).ti,ab

11. 7 or 8 or 9 or 10

12. (prophylactic* or prophylax* or prevent*).ti,ab

13. 11 and 12

\section{Free text search strategy for other databases (Nov 2011)}

(platelet* OR thrombocyte*) AND (transfus* OR infus* OR administ* OR requir*) AND (prophylactic* OR prophylaxis OR prevent OR prevention OR preventing)

\section{MEDLINE \& Embase search strategy (Jan 2002)}

1. Platelet Transfusion.mh.

2. platelet\$ adj10 (substitute $\$$ or transfusion\$ or prophyla \$).tw.

3. 1 or 2

4. haemorrhage.mh.

5. platelet\$.tw.

6. 4 and 5

7. exp Blood Transfusion/ 

8. 5 and 7
9. 3 or 6 or 8

\section{DECLARATIONS OF INTEREST}

Lise Estcourt: author of one of the studies.

Gemma Crighton: none declared.

Simon Stanworth: chief investigator of one of the studies.

Erica Wood: author of one of the studies.

Carolyn Doree: none declared.

Marialena Trivella: none declared.

Sally Hopewell: none declared.

Mike Murphy: author of one the studies.

Alan Tinmouth: none declared.

\section{Additional references}

* Indicates the major publication for the study

BCSH 2003 . British Committee for Standards in Haematology (BCSH). Guidelines for the use of platelet transfusions. British Journal of Haematology. 2003; 122:10-23. [PubMed: 12823341]

BCSH 2004 . British Committee for Standards in Haematology (BCSH). Transfusion guidelines for neonates and older children. British Journal of Haematology. 2004; 124(4):433-53. [PubMed: 14984493]

Benson 2009 . Benson AB, Moss M, Silliman CC. Transfusion-related acute lung injury (TRALI): a clinical review with emphasis on the critically ill. British Journal of Haematology. 2009; 147(4): 431-43. [PubMed: 19663827]

Blajchman 2008 . Blajchman MA, Slichter SJ, Heddle NM, Murphy MF. New strategies for the optimal use of platelet transfusions. Hematology (American Society of Hematology Education Program). 2008:198-204.

Blumberg 2009 . Blumberg N, Spinelli SL, Francis CW, Taubman MB, Phipps RP. The platelet as an immune cell - CD40 ligand and transfusion immune modulation. Immunology Research. 2009; 45:251-60.

Board 2009 . The Board of the German Medical Association on the Recommendation of the Scientific Advisory Board. Platelet concentrates. Cross-sectional guidelines for therapy with blood components and plasma derivatives. Transfusion Medicine and Hemotherapy. 2009; 36:372-82. [PubMed: 21245968]

Bolton-Maggs 2012 . H, Cohen; on behalf of the Serious Hazards of Transfusion (SHOT) Steering Group. The 2011 Annual SHOT Report. Bolton-Maggs, PHB., editor. Serious Hazards of Transfusion (SHOT); 2012.

Burnett 2011 . Burnett AK, Hills RK, Milligan D, Kjeldsen L, Kell J, Russell NH, et al. Identification of patients with acute myeloblastic leukemia who benefit from the addition of gemtuzumab ozogamicin: results of the MRC AML15 trial. Journal of Clinical Oncology. 2011; 29(4):369-77. [PubMed: 21172891] 
Butler 2013 . Butler C, Doree C, Estcourt LJ, Trivella M, Hopewell S, Brunskill SJ, et al. Pathogenreduced platelets for the prevention of bleeding. Cochrane Database of Systematic Reviews. 2013; (3) DOI: 10.1002/14651858.CD009072.

Cameron 2007 . Cameron B, Rock G, Olberg B, Neurath D. Evaluation of platelet transfusion triggers in a tertiary-care hospital. Transfusion. 2007; 47(2):206-11. [PubMed: 17302765]

Cancer Research UK 2013 . Cancer Research UK. [Accessed 14 February 2013] Percentage change in European age-standardised three year average incidence rates, males, UK, 1991-2001 and 2008-2010. Cancer Research UK statistics. http://www.cancerresearchuk.org/cancer-info/ cancerstats/

CDC 2012 . Centers for Disease Control (CDC). United States Cancer Statistics. National Program of Cancer Registries (NPCR); 2012. [Accessed 14 February 2013]

Coleman 2004 . Coleman MP, Rachet B, Woods LM, Mitry E, Riga M, Cooper N, et al. Trends and socioeconomic inequalities in cancer survival in England and Wales up to 2001. British Journal of Cancer. 2004; 90(7):1367-73. [PubMed: 15054456]

Cook 2004 . Cook RJ, Heddle NM, Rebulla P, Sigouin CS, Webert KE. Methods for the analysis of bleeding outcomes in randomized trials of platelet transfusion triggers. Transfusion. 2004; 44:1135-42. [PubMed: 15265116]

De la Serna 2008 . De la Serna J, Montesinos P, Vellenga E, Rayon C, Parody R, Leon A, et al. Causes and prognostic factors of remission induction failure in patients with acute promyelocytic leukemia treated with all-trans retinoic acid and idarubicin. Blood. 2008; 111(7):3395-402. [PubMed: 18195095]

Deeks 2011 . Deeks, JJ.; Higgins, JPT.; Altman, DG.; Higgins, JPT.; Green, S., editors. Cochrane Handbook for Systematic Reviews of Interventions Version 5.1.0 (updated March 2011). The Cochrane Collaboration; 2011. Chapter 9: Analysing data and undertaking meta-analyses. Available from www.cochrane-handbook.org

Duke 1910 . Duke WW. The relation of blood platelets to hemorrhagic disease. Description of a method for determining the bleeding time and coagulation time and report of 3 cases of hemorrhagic disease relieved by transfusion. Journal of the American Medical Association. 1910; 55:1185-92.

Estcourt 2011 . Estcourt LJ, Stanworth SJ, Murphy MF. Platelet transfusions for patients with haematological malignancies: who needs them? British Journal of Haematology. 2011; 154(4): 425-40. [PubMed: 21615375]

Estcourt 2012b . Estcourt LJ, Birchall J, Lowe D, Grant-Casey J, Rowley M, Murphy MF. Platelet transfusions in haematology patients: are we using them appropriately? Vox Sanguinis. 2012; 103(4):284-93. [PubMed: 22775395]

Estcourt 2013a . Estcourt LJ, Heddle N, Kaufman RM, McCullough J, Murphy MF, Slichter S, et al. On behalf of the BEST (Biomedical Excellence for Safer Transfusion) Collaborative. Differences in the methods of assessing and analysing bleeding outcomes in platelet transfusion trials. Transfusion. 2013; 53(7):1531-43. [PubMed: 23305609]

Estcourt 2013b . Estcourt LJ, Pinchon D, Symington E, Kelly AM, Doree C, Brunskill S, et al. Does bleeding affect patient reported outcome measures in patients with myelodysplasia or hematologic malignancies: a systematic review. Transfusion. 2013 [Early on-line publication]. [DOI: $10.1111 /$ trf.12441].

Fielding 2007 . Fielding AK, Richards SM, Chopra R, Lazarus HM, Litzow MR, Buck G, et al. Outcome of 609 adults after relapse of acute lymphoblastic leukemia (ALL); an MRC UKALL12/ECOG 2993 study. Blood. 2007; 109(3):944-50. PUBMED: 17032921. [PubMed: $17032921]$

Friedmann 2002 . Friedmann AM, Sengul H, Lehmann H, Schwartz C, Goodman S. Do basic laboratory tests or clinical observations predict bleeding in thrombocytopenic oncology patients? Transfusion Medicine Reviews. 2002; 16:34-45. [PubMed: 11788928]

Gratwohl 2010 . Gratwohl A, Baldomero H, Aljurf M, Pasquini MC, Bouzas LF, Yoshimi A, et al. Hematopoietic stem cell transplantation: a global perspective. JAMA. 2010; 303(16):1617-24. [PubMed: 20424252] 
Greeno 2007 . Greeno E, McCullough J, Weisdorf D. Platelet utilisation and the transfusion trigger: a prospective analysis. Transfusion. 2007; 72(2):201-5. [PubMed: 17302764]

Heddle 2003 . Heddle NM, Cook RJ, Webert KE, Sigouin C, Rebulla P. Methodologic issues in the use of bleeding as an outcome in transfusion medicine studies. Transfusion. 2003; 43:742-52. [PubMed: 12757525]

Heddle 2008 . Heddle NM, Arnold DM, Boye D, Webert KE, Resz I, Dumont LJ. Comparing the efficacy and safety of apheresis and whole blood-derived platelet transfusions: a systematic review. Transfusion. 2008; 48(7):1447-58. [PubMed: 18482183]

Heddle 2009a . Heddle NM, Cook RJ, Tinmouth A, Kouroukis CT, Hervig T, Klapper E, et al. A randomized controlled trial comparing standard and low dose strategies for transfusion of platelets (SToP) to patients with thrombocytopenia. Blood. 2009; 113(7):1564-73. [PubMed: 19109560]

Heddle 2009b . Heddle, NM.; Webert, K. Investigation of acute transfusion reactions. In: Murphy, MF.; Pamphilion, DH., editors. Practical Transfusion Medicine. 4th Edition. Blackwell; 2009. p. 63-89.

Higby 1974 . Higby DJ, Cohen E, Holland JF, Sinks L. The prophylactic treatment of thrombocytopenic leukemic patients with platelets: a double blind study. Transfusion. 1974; 14:440-5. [PubMed: 4607226]

Higgins 2011a . Higgins, JPT.; Deeks, JJ.; Higgins, JPT.; Green, S., editors. Cochrane Handbook for Systematic Reviews of Interventions Version 5.1.0 (updated March 2011). The Cochrane Collaboration; 2011. Chapter 7: Selecting studies and collecting data. Available from www.cochrane-handbook.org

Higgins 2011b . Higgins, JPT.; Deeks, JJ.; Altman, DG.; Higgins, JPT.; Green, S., editors. Cochrane Handbook for Systematic Reviews of Interventions Version 5.1.0 (updated March 2011). The Cochrane Collaboration; 2011. Chapter 16: Special topics in statistics. Available from www.cochrane-handbook.org

Higgins 2011c . Higgins, JPT.; Altman, DG.; Sterne, JAC.; Higgins, JPT.; Green, S., editors. Cochrane Handbook for Systematic Reviews of Interventions Version 5.1.0 (updated March 2011). The Cochrane Collaboration; 2011. Chapter 8: Assessing risk of bias in included studies. Available from www.cochrane-handbook.org

Knowles 2010 . Cohen, H.; on behalf of the Serious Hazards of Transfusion (SHOT) Steering Group. The 2009 Annual SHOT Report. Knowles, S., editor. Serious Hazards of Transfusion (SHOT); 2010.

Knowles 2011 . Cohen, H.; on behalf of the Serious Hazards of Transfusion (SHOT) Steering Group. The 2010 Annual SHOT Report. Knowles, S., editor. Serious Hazards of Transfusion (SHOT); 2011.

Koreth 2004 . Koreth R, Weinert C, Weisdorf DJ, Key NS. Measurement of bleeding severity: a critical review. Transfusion. 2004; 44:605-17. [PubMed: 15043580]

Lefebvre 2011 . Lefebvre, C.; Manheimer, E.; Glanville, J. Chapter 6: Searching for studies. In: Higgins, JPT.; Green, S., editors. Cochrane Handbook for Systematic Reviews of Interventions Version 5.1.0 (updated March 2011). The Cochrane Collaboration; 2011. Available from www.cochrane-handbook.org

Murphy 1982 . Murphy S, Litwin S, Herring LM, Koch P, Remischovky J, Donaldson MH, et al. Indications for platelet transfusion in children with acute leukemia. American Journal of Hematology. 1982; 12:347-56. [PubMed: 6981349]

NBA 2012 . National Blood Authority. The National Blood Authority's Patient Blood Management Guideline: Module 3 - Medical. National Blood Authority; 2012.

ONS 2012 . ONS. Office of National Statistics. 2012. Cancer incidence and mortality tables and charts. [Accessed 14 February 2013]

Parmar 1998 . Parmar MK, Torri V, Stewart L. Extracting summary statistics to perform metaanalyses of the published literature for survival endpoints. Statistics in Medicine. 1998; 17(24): 2815-34. [PubMed: 9921604] 
Passweg 2012 . Passweg JR, Baldomero H, Gratwohl A, Bregni M, Cesaro S, Dreger P, et al. The EBMT activity survey: 1990-2010. Bone Marrow Transplant. 2012; 47(7):906-23. [PubMed: 22543746]

Patel 2009 . Patel B, Kirkland K, Szydlo R, Pearce R, Clark R, Craddock C, et al. Favorable outcomes with alemtuzumab-conditioned unrelated donor stem cell transplantation in adults with high-risk Philadelphia chromosome-negative acute lymphoblastic leukemia in first complete remission. Haematologica. 2009; 94:1399-406. [PubMed: 19648167]

Pavenski 2013 . Pavenski K, Rebulla P, Duquesnoy R, Saw CL, Slichter SJ, Tanael S, et al. International Collaboration for Guideline Development, Implementation. Evaluation for Transfusion Therapies, Collaborators. Efficacy of HLA-matched platelet transfusions for patients with hypoproliferative thrombocytopenia: a systematic review. Transfusion. 2013 Epub:ahead of print. [DOI: 10.1111/trf.12175].

Pearce 2011 . Pearce S, Rowe GP, Field SP. Screening of platelet for bacterial contamination at the Welsh Blood Service. Transfusion Medicine. 2011; 21(1):25-32. [PubMed: 20854460]

Pendry 2011 . Pendry K, Davies T. An audit of use and wastage in the north west of England and North Wales: where have all the platelets gone? Blood and Transplant Matters. 2011; 34:17-9.

Popovsky 1985 . Popovsky MA, Moore SB. Diagnostic and pathogenetic considerations in transfusion-related acute lung injury. Transfusion. 1985; 25:573-7. [PubMed: 4071603]

Rachet 2009 . Rachet B, Maringe C, Nur U, Quaresma M, Shah A, Woods LM, et al. Populationbased cancer survival trends in England and Wales up to 2007: an assessment of the NHS cancer plan for England. Lancet Oncology. 10(4):351-69.

Review Manager 2012 . The Nordic Cochrane Centre. Review Manager (RevMan). 5.2. Copenhagen: The Cochrane Collaboration; 2012.

Rysler 2010 . Rysler C, Stoffel N, Buser A, Gratwohl A, Tsakiris DA, Stern M. Effect of betablockers, $\mathrm{Ca} 2+$ antagonists, and benzodiazepines on bleeding incidence in patients with chemotherapy induced thrombocytopenia. Platelets. 2010; 21(1):77-83. [PubMed: 19929239]

Schiffer 2001 . Schiffer CA, Anderson KC, Bennett CL, Bernstein S, Elting LS, Goldsmith M, et al. Platelet transfusion for patients with cancer: clinical practice guidelines of the American Society of Clinical Oncology. Journal of Clinical Oncology. 2001; 19:1519-38. [PubMed: 11230498]

Schünemann 2011 . Schünemann, HJ.; Oxman, AD.; Higgins, JPT.; Vist, GE.; Glasziou, P.; Guyatt, GH. Chapter 11: Presenting results and 'Summary of findings' tables. In: Higgins, JPT.; Green, S., editors. Cochrane Handbook for Systematic Reviews of Interventions Version 5.1.0 (updated March 2011). The Cochrane Collaboration; 2011. Available from www.cochrane-handbook.org

Shehata 2009 . Shehata N, Tinmouth A, Naglie G, Freedman J, Wilson K. ABO-identical versus non-identical platelet transfusion: a systematic review. Transfusion. 2009; 49:2442-53. [PubMed: 19903296]

Silliman 2003 . Silliman CC, Boshkov LK, Mehdizadehkashi Z, Elzi DJ, Dickey WO, Podlosky L, et al. Transfusion-related acute lung injury: epidemiology and a prospective analysis of etiologic factors. Blood. 2003; 101(2):454-62. [PubMed: 12393667]

Slichter 1980 . Slichter SJ. Controversies in platelet transfusion therapy. Annual Reviews of Medicine. 1980; 31:509-40.

Slichter 2005 . Slichter SJ, Davis K, Enright H, Braine H, Gernsheimer T, Kao KJ, et al. Factors affecting posttransfusion platelet increments, platelet refractoriness, and platelet transfusion intervals in thrombocytopenic patients. Blood. 2005; 105:4106-14. [PubMed: 15692069]

Slichter 2007 . Slichter SJ. Evidence-based platelet transfusion guidelines. Hematology (American Society of Hematology Education Program). 2007:172-8.

Slichter 2010 . Slichter SJ, Kaufman RM, Assmann SF, McCullough J, Triulzi DJ, Strauss RG, et al. Dose of prophylactic platelet transfusions and prevention of haemorrhage. New England Journal of Medicine. 2010; 362:600-13. [PubMed: 20164484]

Solomon 1978 . Solomon J, Bofenkamp T, Fahey JL, Chillar RK, Beutler E. Platelet prophylaxis in acute non-lymphoblastic leukemia. Lancet. 1978; 1(8058):267. [PubMed: 74683]

Stanworth 2005 . Stanworth SJ, Hyde C, Brunskill S, Murphy MF. Platelet transfusion prophylaxis for patients with haematological malignancies: where to now? British Journal of Haematology. 2005; 131:588-95. [PubMed: 16351634] 
Stanworth 2010 . Stanworth SJ, Dyer C, Choo L, Bakrania L, Copplestone A, Llewelyn C, et al. Do all patients with hematologic malignancies and severe thrombocytopenia need prophylactic platelet transfusions? Background, rationale, and design of a clinical trial (trial of platelet prophylaxis) to assess the effectiveness of prophylactic platelet transfusions. Transfusion Medicine Reviews. 2010; 24(3):163-71. [PubMed: 20656185]

Stanworth 2012 . Stanworth SJ, Estcourt L, Powter G, Kahan BC, Dyer C, Bakrania L, et al. The effect of a no-prophylactic versus prophylactic platelet transfusion strategy on bleeding in patients with hematological malignancies and severe thrombocytopenia (TOPPS trial). A randomized controlled, non-inferiority trial. Blood. 2012; 120(Supplement 1) Abstract 1.

Stanworth 2013 . Stanworth SJ, Estcourt LJ, Powter G, Kahan B, Dyer C, Choo L, et al. A noprophylaxis platelet transfusion strategy for hematologic cancers. New England Journal of Medicine. 2013; 368(19):1771-80. PUBMED: WOS: 000318540000005. [PubMed: 23656642]

Sterne 2011 . Sterne, JAC.; Egger, M.; Moher, D.; Higgins, JPT.; Green, S., editors. Cochrane Handbook for Systematic Reviews of Intervention. Version 5.1.0 (updated March 2011). The Cochrane Collaboration; 2011. Chapter 10: Addressing reporting biases. Available from www.cochrane-handbook.org

Tierney 2007 . Tierney JF, Stewart LA, Ghersi D, Burdett S, Sydes MR. Practical methods for incorporating summary time-to-event data into meta-analysis. Trials. 2007; 8(16) DOI: 10.1186/1745-6215-8-16.

Tinmouth 2007 . Tinmouth, AT. Canadian Blood Services. 4th Edition. Canadian Blood Services; 2007. Chapter 18: Platelet transfusion, alloimmunization and management of platelet refractoriness.

TRAP 1997 . The Trial to Reduce Alloimmunization to Platelets (TRAP) Study Group. Leukocyte reduction and ultraviolet $\mathrm{B}$ irradiation of platelets to prevent alloimmunization and refractoriness to platelet transfusions. New England Journal of Medicine. 1997; 337(26):1861-9. [PubMed: 9417523]

Verma 2009 . Verma A, Agarwal P. Platelet utilization in the developing world: strategies to optimize platelet transfusion practices. Transfusion and Apheresis Science. 2009; 41(2):145-9. [PubMed: 19716339]

Wandt 2012 . Wandt H, Schaefer-Eckart K, Wendelin K, Pilz B, Wilhelm M, Thalheimer M, et al. Therapeutic platelet transfusion versus routine prophylactic transfusion in patients with haematological malignancies: an open-label, multicentre, randomised study. Lancet. 2012; 380(9850):1309-16. [PubMed: 22877506]

WHO 1979 . WHO. WHO Handbook for Reporting Results of Cancer Treatment. World Health Organisation; Geneva: 1979. WHO Offset publication No. 48

\section{References to other published versions of this review}

Estcourt 2012a . Estcourt L, Stanworth SJ, Doree C, Hopewell S, Murphy MF, Tinmouth A, et al. Prophylactic platelet transfusion for prevention of bleeding in patients with haematological disorders after chemotherapy and stem cell transplantation. Cochrane Database of Systematic Reviews. 2012; (5) DOI: 10.1002/14651858.CD004269.pub3.

Stanworth 2004 . Stanworth SJ, Hyde C, Heddle N, Rebulla P, Brunskill S, Murphy MF. Prophylactic platelet transfusion for haemorrhage after chemotherapy and stem cell transplantation. Cochrane Database of Systematic Reviews. 2004; (4) DOI: 10.1002/14651858.CD004269.pub2. 